\title{
Filopodia and their links with membrane traffic and cell adhesion
}

\author{
Jennifer L Gallop \\ Wellcome Trust/CRUK Gurdon Institute and Department of Biochemistry, University \\ of Cambridge, Tennis Court Road, Cambridge, CB2 1QN, UK
}

\begin{abstract}
Filopodia are dynamic, narrow, plasma membrane protrusions filled with bundled actin filaments. Fresh actin monomers are incorporated at the tips furthest away from the cell body, where barbed end polymerases such as formins and Ena/VASP proteins are present in a complex of proteins with other regulatory proteins including Myosin-X. The tightly packed, parallel alignment of actin filaments in the shaft is mediated by bundling, notably by fascin but also fimbrin and some formins. Filopodia often, but need not necessarily, emerge from areas of Arp2/3 complex nucleated F-actin. The membrane surrounding filopodia is tightly curved, where inverse-Bin-Amphiphysin-Rvs proteins deform the membrane, together with Ezrin-Radixin-Moesin proteins. Recent studies have revealed that phosphoinositide metabolism and endophilin-dependent endocytosis are associated with filopodia and that they are a site for virus and exosome internalization. The composition of filopodia can share similarity with the integrin adhesome, and adhesion plays mechanical and tissue matching roles in filopodia function. This review considers how filopodia form and discusses putative mechanistic integration between filopodia, endocytosis and adhesion.
\end{abstract}

(C) 2019. This manuscript version is made available under the CC-BY-NC-ND 4.0 license http://creativecommons.org/licenses/by-nc-nd/4.0/ 


\section{Contents}

1. Introduction

2. Functional roles for filopodia

3. Building filopodia

3.1 Initiation of filopodia formation

3.2 Controversies over the role of the Arp $2 / 3$ complex

3.3 Filopodia tip complex

3.4 Membrane environment

3.5 Bundling actin filaments

3.6 Actin dynamics in filopodia

3.7 Phosphoinositide lipids

4. Filopodia as sites of membrane traffic and adhesion

4.1 Filopodia as locations of endocytosis

4.2 Filopodia as sites of integrin adhesion

5. Conclusions and future directions

Funding

Acknowledgements

References

\section{Introduction}

The ability of cells to sense and move in response to environmental cues underlies the formation of multicellular life. From chemotaxis in Dictyostelium (1) to the network complexity of the human brain (2), orchestration of dynamic actin superstructures is the underlying molecular machinery. Filopodia are finger-like actin-rich protrusions which have become key to understanding how cells transmit and receive messages, and how extracellular signalling and developmental programs construct tissue architecture. They range from 60-200 $\mathrm{nm}$ in diameter and contain 10-30 actin filaments. Filopodia can be just a few microns long or span tens-to-a-hundred microns. There are different kinds of filopodia, and while there are likely to be a selection of different molecular components 
underlying their role and formation, they have attributes in common: long bundled actin filaments that somehow form at a defined location, deform the membrane, intersect with any relevant signalling machinery and dynamically elongate and bundle their actin filaments.

In this review, first I summarize the main functional contexts in which filopodia have been studied. I then aim to draw out common mechanistic principles that operate in filopodia, which arise in different cellular and developmental contexts. In the second part of the review, I discuss the molecules and functional connections between filopodia, endocytosis and integrin-based adhesions. There are situations where membrane traffic and adhesion are coupled to the filopodial machinery and these connections represent an interesting avenue for future research.

\section{Functional roles for filopodia}

During nervous system development, the protrusion of neurites initiates with lamellipodial and filopodial projections, where the actin bundling in filopodia is thought to guide the tracks of microtubules that stabilize the neurite projection $(3,4)$. The neuronal growth cone is the workhorse of axonal guidance and its morphology is characterised by the projection of filopodia, which are used in the response to guidance cues (Figure 1a). Neuronal arborization and synaptogenesis also proceed via filopodia protrusion (4). Synapses emerge from filopodia, which then mature into stabilized connections, with filopodia becoming suppressed over the course of development (Figure 1b) (5). The nervous system has therefore provided multiple lines of investigation as to the roles and molecular mechanisms of filopodia (4).

Similarities have been drawn between neuronal synapses and signalling filopodia that generate morphogen gradients. During early development, specialized filopodia called

cytonemes directly transmit morphogens bidirectionally to and from tissues that signal to each other (6). Cytonemes in the Drosophila airway (Figure 1c) form molecularlycharacterized glutamatergic synapses on their target cells, similar to neuronal synapses, 
where vesicle trafficking co-locates with the cytoneme (7). In early stage Xenopus embryos, membrane trafficking occurs down long filopodia that span across the large blastomeres (8). Cytonemes more generally are thought to have a particular role in propagating morphogen signalling where molecules such as Wnts and Hhs, are lipidmodified (9-11).

Filopodia are also involved during infection, capturing viruses prior to cell entry (12) and propagating them between cells (13). Structures resembling filopodia, called tunnelling nanotubes cross between cells acting as highways transporting prions and organelles (14, 15). Exosomes surf down filopodia and enter cells at hotspots of endocytosis at the base of filopodia, perhaps representing a natural mechanism that is hijacked by pathogens (13, $16)$.

Filopodia play mechanical roles in different types of adhesion processes. The accumulation of myosin- $X$ at tips of filopodia results in the formation of an adhesomelike collection of actin regulatory and mechanosensitive proteins in breast cancer cells (Figure 1d) (17). Filopodia can zipper epithelial sheets together, such as in Drosophila dorsal closure (Figure 1e) (18). Also in Drosophila, cardioblasts express different cell surface adhesion receptors and the accurate cell matching process is mediated by filopodia (19). Development of the tissue relies on these adhesion proteins being functional (19). Filopodial adhesions also join myoblasts together in the initial steps of fusion to form syncytial myotubes during development of adult Drosophila musculature (20). Cadherins can localize to filopodia where they are responsible for force generation, for example, during compaction in the early mouse embryo (21). Filopodia can act as 'sticky fingers' that exert force via adhesive interactions and myosin II activity $(22,23)$. Thus, adhesion is both structurally and functionally integrated with filopodia and their roles in development. 


\section{Building filopodia}

\subsection{Initiation of filopodia formation}

When filopodia emerge from the cell membrane, the prospective tip and base separate and consequently, structures at both the tip and base are implicated in filopodia structure and stabilization. Both changes in membrane curvature and adhesion are observed at filopodial initiation sites. Early timelapse microscopy studies examined sites of filopodia formation in chick neuronal dorsal root ganglion cells, and observed that filopodia initiate with an engorgement of cytoplasm, visible as increased phase density, with the formation of 'nubs' from the membrane (24). Vinculin and phosphotyrosine staining indicated that these nubs were types of adhesions, and that adhesions end up at the tips, in the shaft as well as at the base of resulting filopodia (25). SH2 domain-based probes used in live cell imaging have more recently revealed that phosphotyrosine correlates with forward filopodial movement in cells from Xenopus neural tube explants (26). When viewed by electron microscopy, $\sim 100 \mathrm{~nm}$ focal rings with spokes of actin filaments are seen at the base of filopodia (24). It is not clear how these structures contribute to filopodial assembly as filopodia do not have to form in conjunction with surface adhesion, for example, many can be seen protruding from the upper surface of HeLa cells by lattice light sheet microscopy (27).

According to the convergent elongation model, dense concentrations of actin are suggested to have a role in filopodia formation, which was based on electron microscopy of platinum replicas of B16F1 cell filopodia (28). $\lambda$-shaped bundles of actin appeared to be precursor structures (28) and these have also been seen live using structured illumination microscopy in a glioma-neuronal fusion cell line (29). In hippocampal neurons, filopodia protrude from regions of membrane with both positive and negative membrane curvature that are associated with actin patches (30). Positive (endocytic) membrane curvature is proposed to attract ArhGAP44/Nadrin 2 to abort the initiation of filopodia by Rac GTPase activating protein activity (30), while negative (filopodial) 
membrane curvature is proposed to provide an initial prompt for extension of actin bundles via recruitment of adaptor proteins and actin filament elongators $(31,32)$.

\subsection{Controversies over the role of the Arp2/3 complex}

Actin filaments nucleated by the Arp2/3 complex have been implicated in filopodia formation by several lines of evidence (33). In the convergent elongation model, the barbed ends of actin come together at the tips of the $\lambda$-structure (28). The Arp2/3complex is implicated in nucleating this actin focus, which is then followed by the emergence of an actin bundle via barbed end binding polymerases including the actin elongation factor VASP and bundling protein fascin $(28,34)$. Expression of Diaphanousrelated formin 2 can form filopodia that are embedded in wider actin network (35), though it is also reported that Dia2 filopodia can form separately of the underlying actin network (36).

Despite the Arp2/3 complex generating branched actin filaments, various in vitro models suggest that Arp2/3 complex-generated actin can also give rise to structures similar to filopodia. Lipid membranes can exert forces on Arp2/3 complex-nucleated actin filaments leading to the protrusion of filopodia-like actin bundles into giant unilamellar vesicles in vitro $(37,38)$. In a supported lipid bilayer assay using frog egg extracts, Arp2/3 complex-generated actin is a necessary precursor to the formation of a filopodialike actin bundle where fascin and Dia2 localise independently of a surrounding membrane (39).

Both N-WASP and Scar/WAVE have been suggested as the Arp2/3 complex nucleating promoting factors involved in filopodia. N-WASP was initially implicated in promoting filopodia formation downstream of Cdc $42 \bullet$ GTP from microinjection and biochemical studies (40-42). While filopodia are reduced in response to expression of activated Cdc42 in N-WASP knockout cells (43), some filopodia still form, suggesting the presence of alternative pathways (44). N-WASP overexpression gives rise to filopodia (45), although 
$\mathrm{N}-\mathrm{WASP}$ is also implicated in polymerizing actin during endocytosis, a process of the opposite membrane topology $(46,47)$.

In contrast to N-WASP which is not reported at tips of filopodia, overexpressed and endogenous Scar/WAVE localizes there in mammalian cells and Drosophila (48-50), providing an Arp2/3 activation mechanism separately from Cdc42 $\bullet \mathrm{GTP}$ activation of $\mathrm{N}$ WASP. In Drosophila, Scar/WAVE depletion reduces filopodia formation in BG2 cells, which are thought to be neuronally derived (51). Consistent with a role for Scar/WAVE in filopodia, levels of Rac and Rac•GTP (that co-activates the Scar/WAVE complex (52)) can promote filopodia formation in rat hippocampal neurons (30) and Xenopus embryos (53).

In neuronal systems, both axonal and dendritic filopodia emerge from patches of actin on the neurite shaft, and these are typically Arp2/3 complex-generated (54-56). In cells spreading on fibronectin, the Arp $2 / 3$ complex has been observed in filopodia shafts rather than at filopodia tips (57), where it could allow the protrusion of lamellipodia from or between filopodia downstream of Rac activation (58).

However, contrary to such models, filopodia-like protrusions are still present in the absence of the Arp2/3 complex (59) and an independent filopodia machinery has been proposed (60). Mouse embryonic fibroblasts deficient in ARPC3 have filopodial protrusions, and still move even though they lack the normal lamellipodial architecture (61). In another mouse embryonic fibroblast cell line depleted of the Arp $2 / 3$ complex, long, bundled actin filaments projecting from the cell are still present, and again some degree of cell motility if feasible, while adhesion and cell spreading are reduced (62). There are possible molecular mechanisms of filopodia formation that can give rise to filopodia on Cdc 42 activation independently of the Arp $2 / 3$ complex including the formin $\mathrm{mDia} 2(35,36)$ and phosphorylation of ERM domain proteins (e.g. downstream of sphinosine 1-phosphate $(63,64)$. There could also be an indirect role for the Arp2/3 complex in establishing a high steady state concentration of polymerizable monomers, generated by local recycling, that allow fast elongation of filaments initiated by formins 
once they are activated (65), distinct from the nucleation role suggested by the convergent elongation model. It is also possible that Arp2/3 complex generated actin has mechanical effects in overcoming membrane tension that then would allow filopodial protrusion (38). Significant perturbations, such as deletion of the Arp2/3 complex, are also expected to have indirect effects on the general steady state of actin assembly, which could therefore lead to the production of filopodia-like protrusions in ways that are different from the mechanisms used by cells under normal conditions $(66,67)$. These different possibilities are not mutually exclusive and different types of filopodial protrusion could use different mechanisms (68).

\subsection{Filopodia tip complex}

Filopodia tips are the site of incorporation of new actin monomers that drives the extension of filopodia (69). Formins can both nucleate and elongate actin filaments (70, 71) and they have clear importance in generating and elongating filopodia. Upstream, the small GTPase Rif is implicated in derepressing mDia2 (72). FMNL2 and FMNL3 are also observed at filopodia tips, with a marked reduction in filopodia with knockdown of FMNL3, whereas the roles of FMNL2 are more in lamellipodia $(73,74)$. While filopodia are reduced on knockdown of mDia2, lamellipodia are also affected, so the effect could be indirect (35). In many kinds of filopodia, Ena/VASP family proteins localize to filopodia tips and are important for filopodia formation, notably in cortical neurons preceding neurite formation $(3,75)$ and terminal arborization of Xenopus retinal ganglion cells (76). In B16F1 cells, the knockdown of capping protein leads to a profusion of filopodia which is dependent on the presence of Ena/VASP proteins (77). These filopodia have the usual filopodia markers and the dynamics are indistinguishable from normal filopodia in the cells, suggesting that this is reflecting an endogenous mechanism. Gelsolin, another capper, could not substitute for capping protein (77).

However, a role for Ena/VASP proteins at filopodia tips is not universal, and the effect of removing of Ena/VASP proteins can be restored by mDia2, supporting a primary, direct 
role for mDia2 (3). In U2OS cells, a bone osteosarcoma cell line, where knockdown of Mena (mammalian Enabled) and VASP inhibits filopodia formation, they do not strongly localize to filopodia tips, instead localizing more at adhesions at the base of filopodia. This suggests Ena/VASP proteins may have a role in elongating actin filaments at the early stages of filopodia protrusion rather than in ongoing forward tip movement of already-formed filopodia (74). Instead, FMNL3 is more directly implicated in providing elongation activity (74). In Xenopus retinal ganglion cell growth cones, Xena (Xenopus Enabled) and VASP protein intensity predicts an extension of filopodia but only in a subset of cases (78). In Drosophila D16 cells, out of Ena and Dia, Ena has the more frequent localization at filopodia tips, but is not universally present. Dia can be at the tips or in the shaft and while Dia is the faster elongator and can produce long filopodia, Ena expression counteracts Dia activity (79). Such a mechanism is thought to ensure a balance of long and short filopodia lifetimes generated by the different actin elongation activities of Ena and Dia (79). In fibroblasts lacking mDia2, Ena and VASP, overexpression of constitutively active mDia or of Ena or VASP attracted similar complements of filopodial proteins however their functional properties differed (80).

While work in mouse fibroblasts suggests that there is a stable complex containing VASP at filopodia tips (81), in hippocampal neurons VASP turns over more quickly suggesting a more dynamic tip complex, with the turnover rate dependent on ubiquitination (82). In filopodia-like structures in vitro a semi-dynamic tip complex has given rise to the possibility that different tip complex elongation proteins could exchange with each other. In this model, the integrated outcome of filopodia length dynamics is a result of multiple mechanisms being used in time rather than particular sets of elongators by filopodia type $(50)$.

Myosin-X was identified at filopodia tips in driving the formation of dorsal filopodia that do not adhere to the substratum (83). The motor domain is essential for Myosin-X to drive filopodia whereas the integrin-binding region was dispensible (83). However, when recruited to sites of adhesion, Myosin-X promotes filopodia formation and extension (84). siRNA mediated reduction in the Arp2/3 complex reduces the numbers of myosin-X- 
generated filopodia in COS7 cells, and has a small effect on the length and fraction of long filopodia (84). Myosin-X may therefore act through multiple pathways both dependent and independent of adhesion.

\subsection{Membrane environment}

At the cell membrane, inverse-Bin1-Amphiphysin-Rvs (I-BAR) domain proteins push the membrane outwards and may also keep the high curvature of the membrane through the filopodia shaft (31). One of these proteins, IRSp53, binds activated Cdc42 and VASP thereby linking membrane deformation with signalling to filopodia and elongation of the actin bundle $(32,85,86)$, it is also reported to phase separate along membrane tubules and thereby support negative membrane curvature (87). In the filopodia shaft, ERM domain proteins link the membrane to actin (64), and can work in conjunction with IBAR proteins to stabilize high negative membrane curvature (88). The lipid environment of filopodia appears to be less diffusive than the rest of the plasma membrane (89), which also suggests BAR domain protein involvement as phase separations of $\mathrm{PI}(4,5) \mathrm{P}_{2}$ within the membrane are also implicated in increasing the efficiency of BAR domain protein recruitment (90). $\mathrm{PI}(4,5) \mathrm{P}_{2}$ lipid phase separation also promotes filopodia-like structure formation in cell-free systems $(39,91)$. Inward-facing membrane bending driven by myosin IIB-mediated pulling, is implicated in the suppression of filopodia over the course of neuronal development (30). In microvilli, endocytosis at the base mediated by Pacsin 2 seems to help the membrane fit around the actin bundle to allow correct microvillar morphology (92).

\subsection{Bundling actin filaments}

Closely packed actin filaments are a particular hallmark of filopodia compared to other elongated actin-rich structures (93). The bundler most conventionally associated with filopodia is fascin (94). Fascin has a compact structure that allows it to closely pack the filaments, and knockdown of fascin reduces filopodia numbers $(94,95)$. However, fascin does not function alone; it can be dispensable for filopodia formation, where fimbrin is 
used in conjunction $(96,97)$. Actin filaments are arranged in hexagonal bundles and have a $12 \mathrm{~nm}$ spacing, closest to the packing driven by fimbrin (93). Fimbrin and fascin are proposed to contribute to different stages in the morphology of the bundle with early roles for fimbrin, and role for fascin in giving the straighter, rigid filopodial architecture (97). Fascin binding in filopodia is very dynamic suggesting the opportunity for multiple bundlers to bind at different times (98). Formins are also observed through the shaft of filopodia, for example mDia1 (99) and DAAM1 which is suggested to co-organize actin in the shaft alongside fascin (95).

\subsection{Actin dynamics in filopodia}

Filopodia undergo rapid extension and retraction and can be stabilized in the direction of cell movement and due to adhesion. The incorporation, flow and disassembly of actin in neuronal filopodia has been followed using actin labelled with caged rhodamine derivatives and GFP (69). The protrusion of a filopodium relies on the rate of actin polymerization at the prospective filopodia tip exceeding retrograde flow within its site of emergence. Retrograde flow within filopodia is similar to that in the cell body or growth cone from which filopodia arise, with a high friction state between the filopodial and cortical actin that is dependent on myosin II-mediated pulling of actin filaments away from the membrane $(100,101)$. The influence of mechanical forces on filopodia growth and retraction is linked to adhesion and the composition of both the base and tip of filopodia. Pulling on filopodia tips adhering to carboxylate beads using forces of up to 20 $\mathrm{pN}$ in an optical trap reduced the rates of actin polymerization, leading to retraction driven by retrograde flow (101). Here, the point of weakness was between the tip complex and the membrane. For fibronectin-coated beads, at 10-fold lower forces, filopodia growth is instead promoted and the presence of myosin IIA at the bases of filopodia correlates with increased filopodia lifetime (23). Where adhesion was via lectins and concavalin-A coated beads instead of integrin-mediated adhesion, no stimulatory effect was seen, suggesting communication between the tip and base of filopodia (23). The tips of filopodia are the dominant site where the assembly rate varies and hence is the regulated step in filopodial dynamics, hence the interest in the molecular 
nature of the tip complex (69). Filopodia also move laterally and can rotate and buckle, exerting force as they do so (102). At filopodia tips, the bundled nature of actin filaments can also increase the processivity of Ena/VASP mediated elongation by avidity effects (103). Severing or disassembly of actin filaments is also suggested to occur at the tip during retraction, via fascin-mediated stimulation of cofilin (104).

\subsection{Phosphoinositide lipids}

Phosphoinositide lipids are involved in many cell biological processes at the plasma membrane and filopodia join that list. $\mathrm{PI}(4,5) \mathrm{P}_{2}$, the major hallmark phosphoinositide lipid of the plasma membrane causes the nucleation of actin at filopodia-like structures (39) and is a substrate for production of $\mathrm{PI}(3,4,5) \mathrm{P}_{3}$, which is implicated in filopodia formation in diverse contexts (105). Filopodia arise from regions of $\mathrm{PI}(3,4,5) \mathrm{P}_{3}$ enrichment in neurons (106), likely via the formation an initial actin patch, rather than a role of $\mathrm{PI}(3,4,5) \mathrm{P}_{3}$ in the elongation of the bundle (107). Filopodia numbers are reduced in phosphoinositide 3-kinase (PI3K) mutant Drosophila embryos, and in embryos overexpressing PTEN, leading to a reduced zippering speed during dorsal closure (108). In Caenorhabditis elegans HSN neurons that migrate in response to netrin, both PI3K (unc-34) and lamellipodin (mig-10) mutants have reduced filopodia (109). As well as $\mathrm{PI}(3,4,5) \mathrm{P}_{3}$ being implicated in Myosin-X function $(110), \mathrm{PI}(3,4) \mathrm{P}_{2}$ is also downstream of $\mathrm{PI} 3 \mathrm{~K}$ and is recently suggested to have a role in filopodia (17).

\section{Filopodia as sites of membrane traffic and adhesion}

Recent publications have highlighted that not only are filopodia linked to lamellipodia, as has long been appreciated, but also to membrane traffic and adhesion. $\lambda$-structures of actin are sites for the budding of endocytic vesicles which bud via clathrin-independent pathways, and traffic into the cell on actin bundles (29). Increased plasma membrane signal that may correspond to an endocytosing vesicle, has been suggested at the tips of Myosin-X filopodia (17). Some sort of coupling between invagination and protrusion might help in regulating filopodial dynamics either at initiation or on an ongoing basis 
(Figure 2). Adhesions, endocytic vesicles and the tips of filopodia all similarly use phosphoinositide lipids to recruit actin regulatory proteins at the inner surface of the plasma membrane. Are these entirely separate processes? Or is a nascent adhesion or budding endocytic vesicle coordinated with the formation of a filopodium (Figure 2a)? Can membrane traffic or adhesion within a filopodium determine its length, growth, retraction or persistence time in conjunction with actin dynamics (Figure 2b)? Any answers to these questions remain speculative and are an important avenue of future research in understanding filopodia because of their mechanical and signalling roles.

\subsection{Filopodia as locations of endocytosis}

The budding of vesicles in filopodia (111), endocytosis associated with actin bundles (29), and viruses hijacking filopodia for capture and internalization $(12,16)$ provide evidence that endocytosis occurs at filopodia. Moreover, inhibitors of endocytosis including K44A dynamin, Eps15 DN mutant, or RNAi of clathrin heavy chain all cause a marked reduction in filopodia $(29,45)$, as does acute treatment with the small molecule dynamin inhibitor, dynasore $(112,113)$. These interventions would also cause other changes in membrane dynamics or direct actin-related roles of dynamin rather than endocytic roles, so their effects could be indirect (114). Dynasore also has non-specific effects, shown by comparison to dynamin 1,2, 3 triple knockout cells (115), so it may be that endocytosis is not being directly targeted in this situation.

The maturation of filopodia into sites of synaptic vesicle recycling and activity-dependent neuronal development makes it enticing to think there may be a direct mechanistic coupling $(4,116)$. Indeed, synaptotagmin has been suggested as a molecular link: when it is overexpressed in fibroblasts, many filopodia are formed, and the active region has been localized to a positively charged region of the protein $(117,118)$. Endophilin is another possible molecular link: it is involved in both fast synaptic vesicle endocytosis and vesicle budding at filopodia $(29,119)$. In retinal ganglion cell axonal arbors of the zebrafish optic tectum, new branches form at synaptic regions (116), where filopodia 
move forward, extend and retract back from synaptic sites. Thus, proteins associated with synaptic vesicles are both found associated with filopodia and influence their formation.

Endocytosis in the Wnt pathway has been linked to filopodia during Wnt treatment. Endogenous endocytic adaptor protein 2 (AP-2) complexes are observed at filopodia tips in dorsal spinal commissural neurons, and Frizzled 3 and AP-2 colocalize there and are internalized while trafficking inwards on filopodia in the presence of Wnt (111). Neuronal growth cones could use endocytosis to bring in information from the environment, activating actin polymerization and causing forward movement of filopodia (111).

In Drosophila dorsal closure and wound healing, filopodia drive the adhesion and zippering of the cell sheets $(18,108)$. The actin cable that closes the edges of the wound is connected from cell to cell via adherens junctions. At the beginning of wound healing, dynamin and clathrin accumulate in spots at the wound edge colocalizing with the actin puncta, which are reduced with dynamin mutant shibire and dominant negative Rab5, suggesting that endocytosis of the junctions results in subsequent stimulation of protrusions (120).

A link between endocytosis and filopodia has been observed with myosin VI. When a chimeric myosin is made converting a normally minus-end directed motor myosin VI to a plus-end directed motor retaining cargo-interacting capabilities, cultured cells form many actin-rich filopodia in a similar way to myosin-X overexpression (121). These filopodia were not dependent on actin nucleation by the Arp2/3 complex or Drf3/mDia2 formin and had endosomes at their base (121).

Thus, diverse examples from different organisms and cellular systems are suggestive of a an underlying coordination between vesicle trafficking and filopodia. 


\subsection{Filopodia as locations of integrin adhesion}

Integrin-based adhesions can be located at filopodia tips, shafts and in basal adhesions, and are important for guidance and signalling. Myosin-X expression recruits binding partners including $\beta 1$-integrin and vinculin to filopodia tips in cancer cells supporting their formation and elongation (84). $\beta 1$-integrin and vinculin knockdown have no effect on numbers of Myosin-X filopodia, but decrease the fraction of long filopodia and the length of filopodia. Arp2/3 complex and $\beta 1$-integrin accumulate where filopodia change direction (84), suggesting that adhesions are 'decision points' for filopodia.

In growth cones and endothelial cell branching in angiogenesis, point contacts of focal adhesions in filopodia are proposed to be part of an integrated machinery used for branching (122). VASP, which localizes to both filopodia and adhesions is proposed to help form a filopodial adhesion which then propagades down the filopodium by retrograde flow, adhering the filopodia site to the substrate (122).

MDA-MB-231 cells grown on fibronectin (Figure 1c) are a model for adhesive filopodia in breast cancer, where Myosin-X is upregulated (123). Talin is recruited to these filopodia while phosphoinositide 3-kinase and L-type calcium channel blockers inhibit their formation. Calcium channels localize to the tips of filopodia which are linked to adhesion, as activating $\beta 1$-integrin increases filopodia numbers and integrin inside-out signalling triggers Src activation and calcium release (123).

A selection of integrin adhesion-associated proteins that are present in filopodia has recently been extensively visually mapped by structured illumination microscopy (17). Filopodia are regions of low force, so do not contain mature adhesions and this was reflected in the subset of core adhesome proteins found in filopodia - the so-called 'filopodome' (17). As filopodia stabilize, more proteins, such as paxillin, were increasingly localized to them. As lamellipodia advance, filopodial adhesions triggered the formation of nascent adhesions. VASP and talin were found in most filopodia together with FMNL3, lamellipodin, mDia2 and fascin $(17,22)$. p130Cas/BCAR1 was 
identified as a mechanosensitive protein that localizes to filopodia. It is phosphorylated by Src in response to mechanical stretch (124). p130Cas/BCAR1 is used by filopodia to sense the stiffness of the substrate, attenuating new filopodia downstream of stabilization (17).

In myotube filopodia in Drosophila, which are used for navigation towards tendon cells in the developing musculature, integrins are present in filopodia though do not form mature adhesive structures (125). Although the suppression of filopodia occurs contemporaneously with formation of adhesion between tendon cells and the myotube, in these cells integrin signalling suppresses filopodia through adaptor protein Git and cytoskeletal protein kinase Pak rather than stabilising or promoting filopodia through adhesion (125).

Thus, while the association of adhesions and filopodia is variable and not obligatory, adhesion has clear functional and possibly also direct mechanistic roles in establishing filopodia, at least in 2D cell culture.

\section{Conclusions and future directions}

After this list of situations with possible coincidences betwen the endocytic and adhesive machinery with filopodia, what might any function of a coordination be? Adhesions could nucleate sufficient actin to sprout filopodia to aid in mechanical sensing. An endocytic burst of actin could supply a focus of branched actin to indicate the site of receptor activation and a need for the elongation of a filopodium as a specialised signalling compartment (Figure 2a). A coupling between exo- and endocytosis and filopodia could supply membrane to these narrow protrusions and help provide the high membrane curvature and dynamics necessary for filopodial protrusion and retraction. Adhesion could be communicating with the actin machinery to influence the rates of filopodia growth and adhesion (Figure 2b). Perhaps an integration of membrane dynamics and adhesion with filopodia machinery would contribute to regulation of filopodia length and lifetime. 
Recent studies that give rise to hypotheses about vesicle trafficking and adhesive links to filopodia both rely on superresolution techniques because of the small size of filopodia $(17,29)$. The ability to do live superresolution microscopy will be a big advance to study how endocytic and adhesive remodelling relates to intense dynamics often displayed by filopodia in migrating cells. The ability to image in multiple channels simultaneously, with a known array of markers for endocytic and adhesive structures will also be necessary to further test how adhesion and membrane traffic integrate with filopodia.

How filopodia, cytonemes, tunnelling nanotubes and microspikes are similar or distinct from each other needs further research so that we can accurately compare related structures. Tracking endogenous protein composition via CRISPR/Cas9 knock-in technology in native cellular filopodia will help, as overexpression studies as well as deletion studies are problematic for their degree of invasiveness. For adhesion, glassbottom dishes represent one extreme of ECM and mechanical environment. Tissue imaging studies using organoids and embryos will be necessary to understand how actin regulation at adhesions, during endocytosis and at filopodia are employed in cells during the course of development.

\section{Funding}

JLG's work has been supported by a Wellcome Research Career Development Fellowship WT095829AI, the European Research Council Grant 281971 and Issac Newton Trust grants 11.06(v) and 18.23(j). The Gurdon Institute receives core grants from Cancer Research UK (C6946/A14492) and Wellcome (092096).

\section{Acknowledgements}

Thank you to Bishara Marzook, Yvonne Vallis and Julia Mason for reading and editing the manuscript. 
(a) Xenopus retinal ganglion cell growth cone

(b) Dendritic filopodia in rat hippocampal neurons

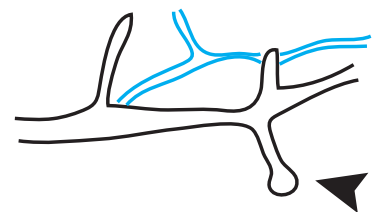

(d) Human breast cancer cell

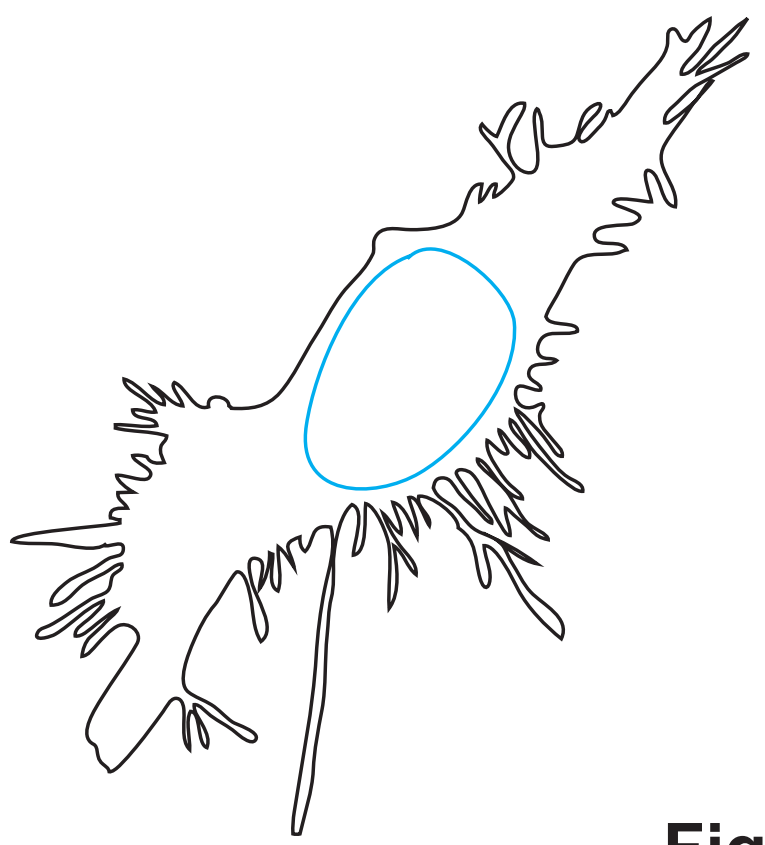

(c) Drosophila air sac primordium

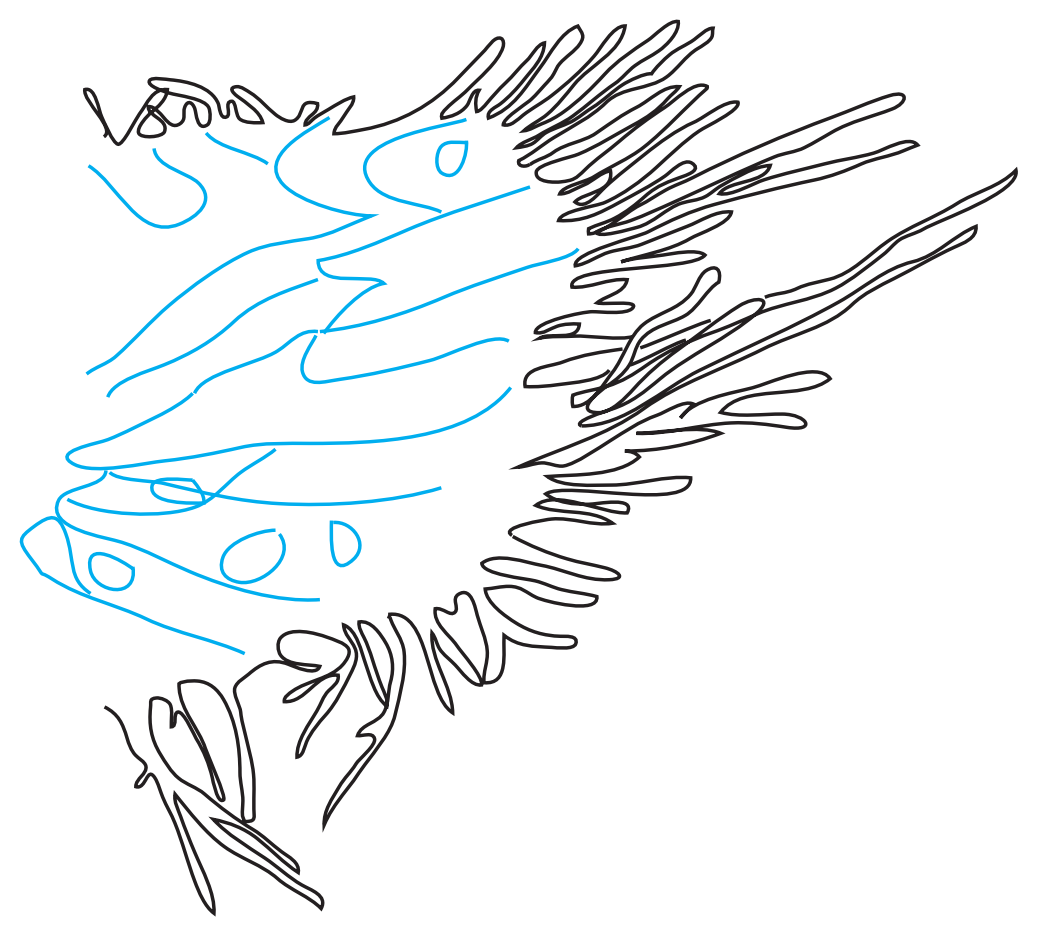

(e) Drosophila dorsal closure leading edge

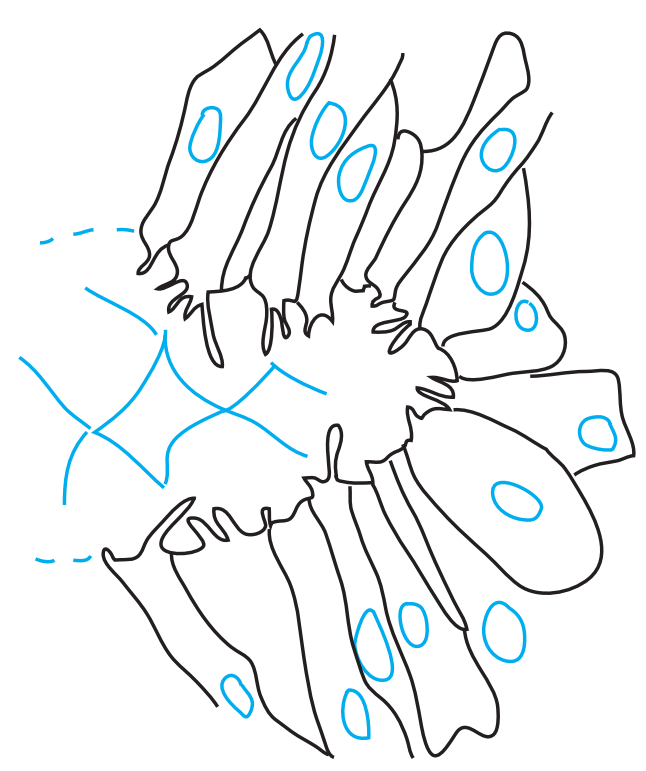

Figure 1 

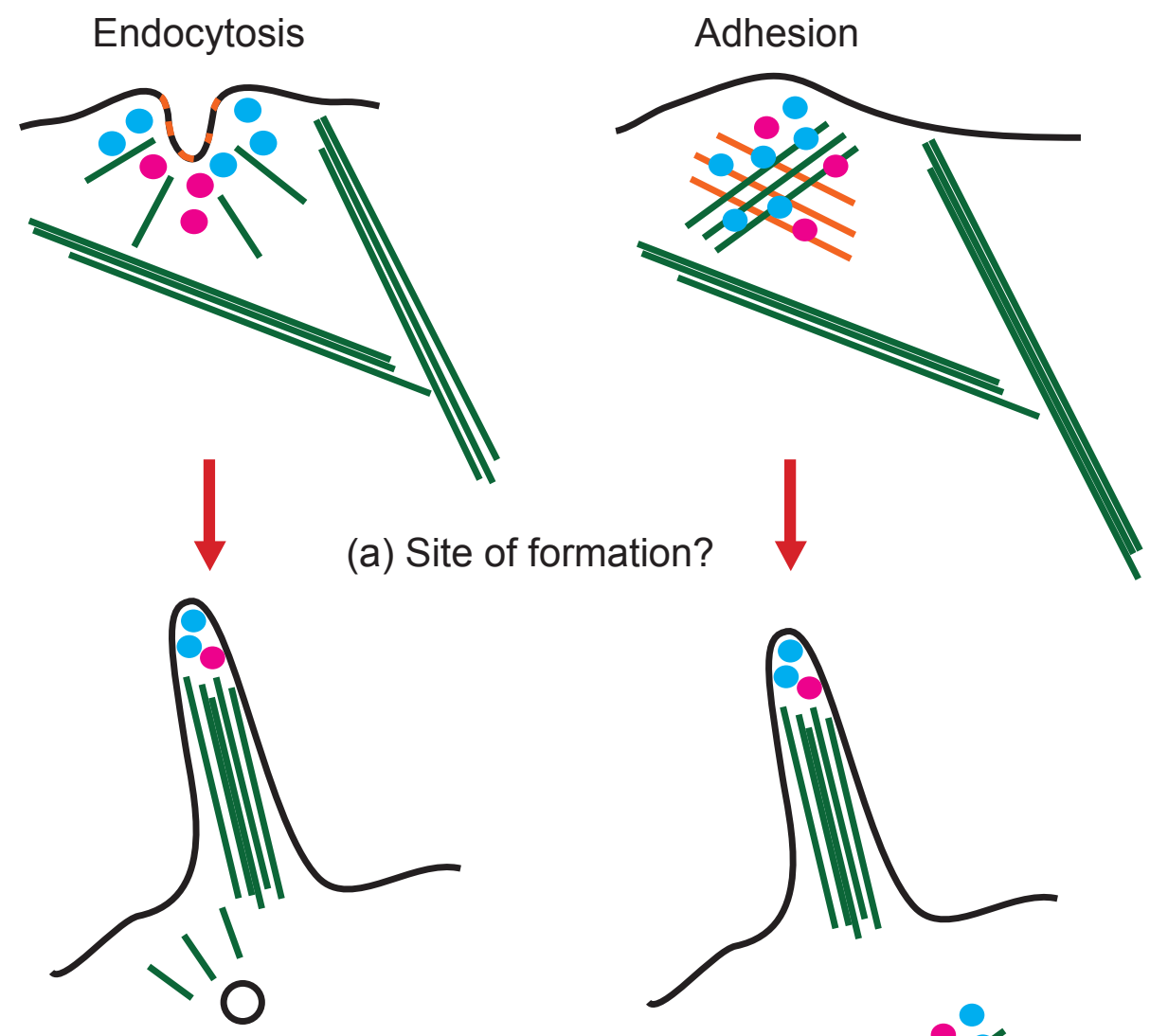

$\checkmark$

(b) Length and dynamics?
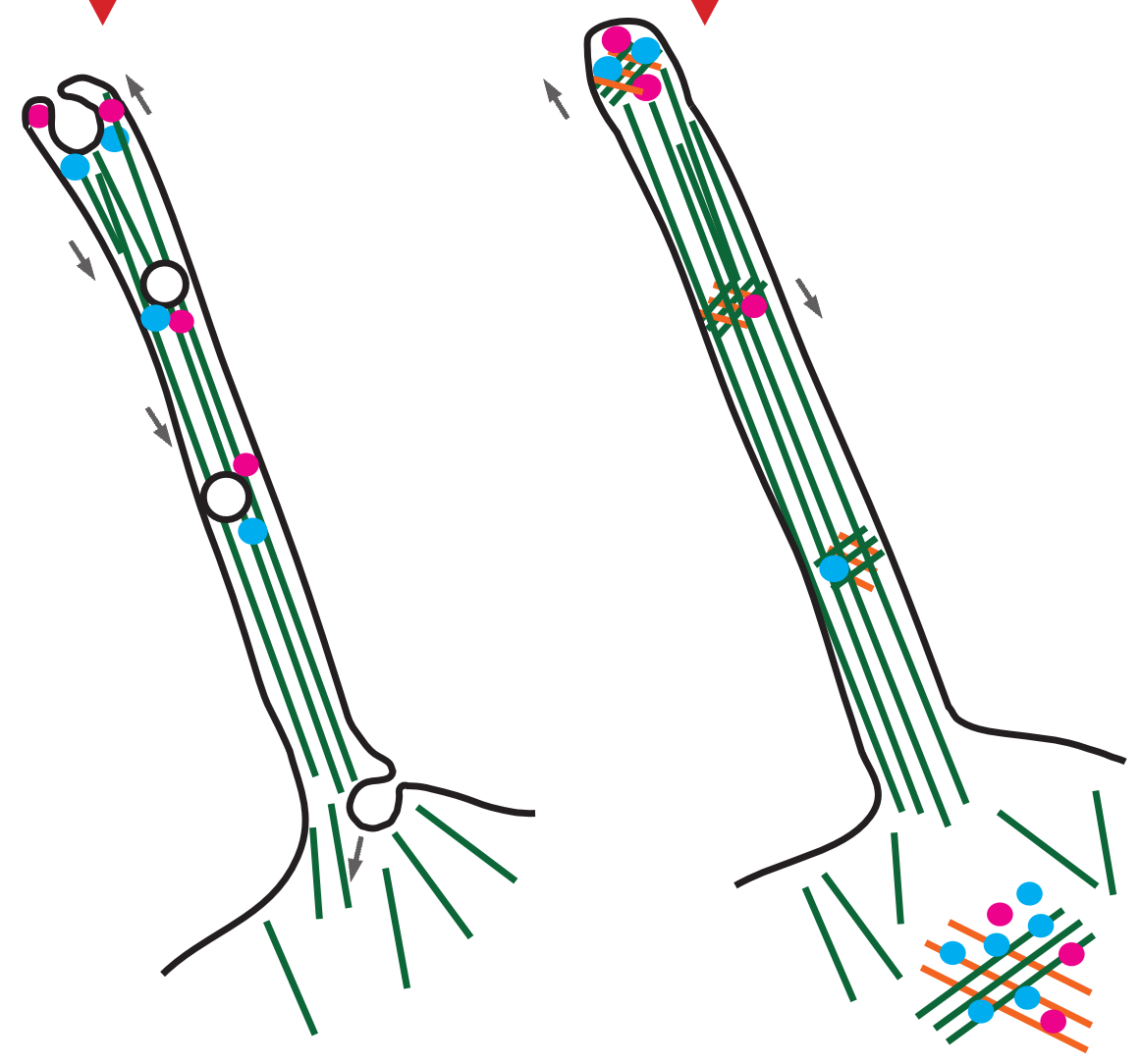

Figure 2 


\section{Figure Captions}

Figure 1 Filopodia in different developmental contexts (a) neuronal growth cones, where filopodia accompany axonal pathfinding (b) dendritic filopodia that contact axons and are precursors to dendritic spines (arrow), blue indicates an axon (c) Cytonemes in the Drosophila air sac primordium, blue indicates nuclear and plasma membranes within the tissue (d) filopodia of MDA-MBA-231 cells showing nuclear membrane in blue (e) filopodia from leading edge cells in Drosophila dorsal closure, nuclear and amnioserosa cell plasma membranes in blue. Images are sketches based on micrographs and shown at equivalent sizes.

\section{Figure 2 Thought-provoking models for endocytosis and adhesion in filopodia}

growth (a) Sites of endocytosis and adhesion coincide with filopodia, so are they involved in determining where a filopodium forms? (b) Membrane turnover and effects of adhesion can tune actin dynamics and the persistence of filopodia so are endocytosis and adhesion determining filopodial length and lifetime $?$ Green = actin filaments, blue and magenta and actin regulatory proteins, orange $=$ transmembrane receptors.

\section{References}

1. Nichols JM, Veltman D, Kay RR. Chemotaxis of a model organism: progress with Dictyostelium. Current opinion in cell biology. 2015;36:7-12.

2. Menon S, Gupton SL. Building Blocks of Functioning Brain: Cytoskeletal Dynamics in Neuronal Development. International review of cell and molecular biology. 2016;322:183-245.

3. Dent EW, Kwiatkowski AV, Mebane LM, Philippar U, Barzik M, Rubinson DA, Gupton S, Van Veen JE, Furman C, Zhang J, Alberts AS, Mori S, Gertler FB. Filopodia are required for cortical neurite initiation. Nature cell biology. 2007;9(12):1347-59.

4. Gallo G. Mechanisms underlying the initiation and dynamics of neuronal filopodia: from neurite formation to synaptogenesis. International review of cell and molecular biology. 2013;301:95-156.

5. Ziv NE, Smith SJ. Evidence for a role of dendritic filopodia in synaptogenesis and spine formation. Neuron. 1996;17(1):91-102.

6. Kornberg TB. Distributing signaling proteins in space and time: the province of cytonemes. Current opinion in genetics \& development. 2017;45:22-7. 
7. Huang H, Liu S, Kornberg TB. Glutamate signaling at cytoneme synapses. Science. 2019;363(6430):948-55.

8. Danilchik M, Williams M, Brown E. Blastocoel-spanning filopodia in cleavagestage Xenopus laevis: Potential roles in morphogen distribution and detection. Developmental biology. 2013;382(1):70-81.

9. Stanganello E, Hagemann AI, Mattes B, Sinner C, Meyen D, Weber S, Schug A, Raz E, Scholpp S. Filopodia-based Wnt transport during vertebrate tissue patterning. Nature communications. 2015;6:5846.

10. Sanders TA, Llagostera E, Barna M. Specialized filopodia direct long-range transport of SHH during vertebrate tissue patterning. Nature. 2013;497(7451):628-32.

11. Gradilla AC, Guerrero I. Cytoneme-mediated cell-to-cell signaling during development. Cell and tissue research. 2013;352(1):59-66.

12. Chang K, Baginski J, Hassan SF, Volin M, Shukla D, Tiwari V. Filopodia and Viruses: An Analysis of Membrane Processes in Entry Mechanisms. Frontiers in microbiology. 2016;7:300.

13. Aggarwal A, Iemma TL, Shih I, Newsome TP, McAllery S, Cunningham AL, Turville SG. Mobilization of HIV spread by diaphanous 2 dependent filopodia in infected dendritic cells. PLoS pathogens. 2012;8(6):e1002762.

14. Gousset K, Schiff E, Langevin C, Marijanovic Z, Caputo A, Browman DT, Chenouard N, de Chaumont F, Martino A, Enninga J, Olivo-Marin JC, Mannel D, Zurzolo C. Prions hijack tunnelling nanotubes for intercellular spread. Nature cell biology. 2009;11(3):328-36.

15. Rustom A, Saffrich R, Markovic I, Walther P, Gerdes HH. Nanotubular highways for intercellular organelle transport. Science. 2004;303(5660):1007-10.

16. Heusermann W, Hean J, Trojer D, Steib E, von Bueren S, Graff-Meyer A, Genoud C, Martin K, Pizzato N, Voshol J, Morrissey DV, Andaloussi SE, Wood MJ, Meisner-Kober NC. Exosomes surf on filopodia to enter cells at endocytic hot spots, traffic within endosomes, and are targeted to the ER. The Journal of cell biology. 2016;213(2):173-84.

17. Jacquemet G, Stubb A, Saup R, Miihkinen M, Kremneva E, Hamidi H, Ivaska J. Filopodome Mapping Identifies p130Cas as a Mechanosensitive Regulator of Filopodia Stability. Current biology : CB. 2019;29(2):202-16 e7.

18. Jacinto A, Wood W, Balayo T, Turmaine M, Martinez-Arias A, Martin P. Dynamic actin-based epithelial adhesion and cell matching during Drosophila dorsal closure. Current biology : CB. 2000;10(22):1420-6.

19. Zhang S, Amourda C, Garfield D, Saunders TE. Selective Filopodia Adhesion Ensures Robust Cell Matching in the Drosophila Heart. Developmental cell. 2018;46(2):189-203 e4.

20. Segal D, Dhanyasi N, Schejter ED, Shilo BZ. Adhesion and Fusion of Muscle Cells Are Promoted by Filopodia. Developmental cell. 2016;38(3):291-304.

21. Fierro-Gonzalez JC, White MD, Silva JC, Plachta N. Cadherin-dependent filopodia control preimplantation embryo compaction. Nature cell biology. 2013;15(12):1424-33.

22. Lagarrigue F, Vikas Anekal P, Lee HS, Bachir AI, Ablack JN, Horwitz AF, Ginsberg MH. A RIAM/lamellipodin-talin-integrin complex forms the tip of sticky fingers that guide cell migration. Nature communications. 2015;6:8492. 
23. Alieva NO, Efremov AK, Hu S, Oh D, Chen Z, Natarajan M, Ong HT, Jegou A, Romet-Lemonne G, Groves JT, Sheetz MP, Yan J, Bershadsky AD. Myosin IIA and formin dependent mechanosensitivity of filopodia adhesion. Nature communications. 2019;10(1):3593.

24. Steketee M, Balazovich K, Tosney KW. Filopodial initiation and a novel filament-organizing center, the focal ring. Molecular biology of the cell. 2001;12(8):2378-95.

25. Steketee MB, Tosney KW. Three functionally distinct adhesions in filopodia: shaft adhesions control lamellar extension. The Journal of neuroscience : the official journal of the Society for Neuroscience. 2002;22(18):8071-83.

26. Robles E, Woo S, Gomez TM. Src-dependent tyrosine phosphorylation at the tips of growth cone filopodia promotes extension. The Journal of neuroscience : the official journal of the Society for Neuroscience. 2005;25(33):7669-81.

27. Chen BC, Legant WR, Wang K, Shao L, Milkie DE, Davidson MW, Janetopoulos C, Wu XS, Hammer JA, 3rd, Liu Z, English BP, Mimori-Kiyosue Y, Romero DP, Ritter AT, Lippincott-Schwartz J, Fritz-Laylin L, Mullins RD, Mitchell DM, Bembenek JN, Reymann AC, Bohme R, Grill SW, Wang JT, Seydoux G, Tulu US, Kiehart DP, Betzig E. Lattice light-sheet microscopy: imaging molecules to embryos at high spatiotemporal resolution. Science. 2014;346(6208):1257998.

28. Svitkina TM, Bulanova EA, Chaga OY, Vignjevic DM, Kojima S, Vasiliev JM, Borisy GG. Mechanism of filopodia initiation by reorganization of a dendritic network. The Journal of cell biology. 2003;160(3):409-21.

29. Nozumi M, Nakatsu F, Katoh K, Igarashi M. Coordinated Movement of Vesicles and Actin Bundles during Nerve Growth Revealed by Superresolution Microscopy. Cell reports. 2017;18(9):2203-16.

30. Galic M, Tsai FC, Collins SR, Matis M, Bandara S, Meyer T. Dynamic recruitment of the curvature-sensitive protein ArhGAP44 to nanoscale membrane deformations limits exploratory filopodia initiation in neurons. eLife. 2014;3:e03116. 31. Mattila PK, Pykalainen A, Saarikangas J, Paavilainen VO, Vihinen H, Jokitalo E, Lappalainen P. Missing-in-metastasis and IRSp53 deform PI(4,5)P2-rich membranes by an inverse BAR domain-like mechanism. The Journal of cell biology. 2007;176(7):95364.

32. Saarikangas J, Zhao H, Pykalainen A, Laurinmaki P, Mattila PK, Kinnunen PK, Butcher SJ, Lappalainen P. Molecular mechanisms of membrane deformation by I-BAR domain proteins. Current biology : CB. 2009;19(2):95-107.

33. Yang C, Svitkina T. Filopodia initiation: focus on the Arp $2 / 3$ complex and formins. Cell adhesion \& migration. 2011;5(5):402-8.

34. Vignjevic D, Yarar D, Welch MD, Peloquin J, Svitkina T, Borisy GG. Formation of filopodia-like bundles in vitro from a dendritic network. The Journal of cell biology. 2003;160(6):951-62.

35. Yang C, Czech L, Gerboth S, Kojima S, Scita G, Svitkina T. Novel roles of formin $\mathrm{mDia} 2$ in lamellipodia and filopodia formation in motile cells. PLoS biology. 2007;5(11):e317.

36. Block J, Stradal TE, Hanisch J, Geffers R, Kostler SA, Urban E, Small JV, Rottner K, Faix J. Filopodia formation induced by active mDia2/Drf3. Journal of microscopy. 2008;231(3):506-17. 
37. Liu AP, Richmond DL, Maibaum L, Pronk S, Geissler PL, Fletcher DA. Membrane-induced bundling of actin filaments. Nat Phys. 2008;4:789-93.

38. Simon C, Kusters R, Caorsi V, Allard A, Abou-Ghali M, Manzi J, Di Cicco A, Levy D, Lenz M, Joanny J-F, Campillo C, Plastino J, Sens P, Sykes C. Actin dynamics drive cell-like membrane deformation. Nature Physics. 2019; doi.org/10.1038/s41567019-0464-1.

39. Lee K, Gallop JL, Rambani K, Kirschner MW. Self-assembly of filopodia-like structures on supported lipid bilayers. Science. 2010;329(5997):1341-5.

40. Nobes CD, Hall A. Rho, rac, and cdc42 GTPases regulate the assembly of multimolecular focal complexes associated with actin stress fibers, lamellipodia, and filopodia. Cell. 1995;81(1):53-62.

41. Ma L, Cantley LC, Janmey PA, Kirschner MW. Corequirement of specific phosphoinositides and small GTP-binding protein Cdc42 in inducing actin assembly in Xenopus egg extracts. The Journal of cell biology. 1998;140(5):1125-36.

42. Rohatgi R, Ma L, Miki H, Lopez M, Kirchhausen T, Takenawa T, Kirschner MW. The interaction between N-WASP and the Arp2/3 complex links Cdc42-dependent signals to actin assembly. Cell. 1999;97(2):221-31.

43. Snapper SB, Takeshima F, Anton I, Liu CH, Thomas SM, Nguyen D, Dudley D, Fraser H, Purich D, Lopez-Ilasaca M, Klein C, Davidson L, Bronson R, Mulligan RC, Southwick F, Geha R, Goldberg MB, Rosen FS, Hartwig JH, Alt FW. N-WASP deficiency reveals distinct pathways for cell surface projections and microbial actin-based motility. Nature cell biology. 2001;3(10):897-904.

44. Lommel S, Benesch S, Rottner K, Franz T, Wehland J, Kuhn R. Actin pedestal formation by enteropathogenic Escherichia coli and intracellular motility of Shigella flexneri are abolished in N-WASP-defective cells. EMBO reports. 2001;2(9):850-7. 45. Bu W, Chou AM, Lim KB, Sudhaharan T, Ahmed S. The Toca-1-N-WASP complex links filopodial formation to endocytosis. The Journal of biological chemistry. 2009;284(17):11622-36.

46. Merrifield CJ, Qualmann B, Kessels MM, Almers W. Neural Wiskott Aldrich Syndrome Protein (N-WASP) and the Arp2/3 complex are recruited to sites of clathrinmediated endocytosis in cultured fibroblasts. European journal of cell biology. 2004;83(1):13-8.

47. Kessels MM, Qualmann B. Syndapins integrate N-WASP in receptor-mediated endocytosis. The EMBO journal. 2002;21(22):6083-94.

48. Hahne P, Sechi A, Benesch S, Small JV. Scar/WAVE is localised at the tips of protruding lamellipodia in living cells. FEBS letters. 2001;492(3):215-20.

49. Nozumi M, Nakagawa H, Miki H, Takenawa T, Miyamoto S. Differential localization of WAVE isoforms in filopodia and lamellipodia of the neuronal growth cone. Journal of cell science. 2003;116(Pt 2):239-46.

50. Dobramysl U, Jarsch IK, Shimo H, Inoue Y, Richier B, Gadsby JR, Mason J, Walrant A, Butler R, Hannezo E, Simons BD, Gallop JL. Constrained actin dynamics emerges from variable compositions of actin regulatory protein complexes. BioRxiv. 2019;525725.

51. Biyasheva A, Svitkina T, Kunda P, Baum B, Borisy G. Cascade pathway of filopodia formation downstream of SCAR. Journal of cell science. 2004;117(Pt 6):837-48. 
52. Lebensohn AM, Kirschner MW. Activation of the WAVE complex by coincident signals controls actin assembly. Molecular cell. 2009;36(3):512-24.

53. Tahinci E, Symes K. Distinct functions of Rho and Rac are required for convergent extension during Xenopus gastrulation. Developmental biology. 2003;259(2):318-35.

54. Korobova F, Svitkina T. Arp2/3 complex is important for filopodia formation, growth cone motility, and neuritogenesis in neuronal cells. Molecular biology of the cell. 2008;19(4):1561-74.

55. Spillane M, Ketschek A, Jones SL, Korobova F, Marsick B, Lanier L, Svitkina T, Gallo G. The actin nucleating Arp2/3 complex contributes to the formation of axonal filopodia and branches through the regulation of actin patch precursors to filopodia. Developmental neurobiology. 2011;71(9):747-58.

56. Korobova F, Svitkina T. Molecular architecture of synaptic actin cytoskeleton in hippocampal neurons reveals a mechanism of dendritic spine morphogenesis. Molecular biology of the cell. 2010;21(1):165-76.

57. Johnston SA, Bramble JP, Yeung CL, Mendes PM, Machesky LM. Arp2/3 complex activity in filopodia of spreading cells. BMC cell biology. 2008;9:65.

58. Guillou H, Depraz-Depland A, Planus E, Vianay B, Chaussy J, Grichine A, Albiges-Rizo C, Block MR. Lamellipodia nucleation by filopodia depends on integrin occupancy and downstream Rac1 signaling. Experimental cell research. 2008;314(3):478-88.

59. Steffen A, Faix J, Resch GP, Linkner J, Wehland J, Small JV, Rottner K, Stradal TE. Filopodia formation in the absence of functional WAVE- and Arp2/3-complexes. Molecular biology of the cell. 2006;17(6):2581-91.

60. Faix J, Breitsprecher D, Stradal TE, Rottner K. Filopodia: Complex models for simple rods. The international journal of biochemistry \& cell biology. 2009;41(89):1656-64.

61. Suraneni P, Rubinstein B, Unruh JR, Durnin M, Hanein D, Li R. The Arp2/3 complex is required for lamellipodia extension and directional fibroblast cell migration. The Journal of cell biology. 2012;197(2):239-51.

62. Wu C, Asokan SB, Berginski ME, Haynes EM, Sharpless NE, Griffith JD, Gomez $\mathrm{SM}$, Bear JE. Arp2/3 is critical for lamellipodia and response to extracellular matrix cues but is dispensable for chemotaxis. Cell. 2012;148(5):973-87.

63. Nakamura N, Oshiro N, Fukata Y, Amano M, Fukata M, Kuroda S, Matsuura Y, Leung T, Lim L, Kaibuchi K. Phosphorylation of ERM proteins at filopodia induced by Cdc42. Genes to cells : devoted to molecular \& cellular mechanisms. 2000;5(7):571-81. 64. Gandy KA, Canals D, Adada M, Wada M, Roddy P, Snider AJ, Hannun YA, Obeid LM. Sphingosine 1-phosphate induces filopodia formation through S1PR2 activation of ERM proteins. The Biochemical journal. 2013;449(3):661-72.

65. Carlier MF, Shekhar S. Global treadmilling coordinates actin turnover and controls the size of actin networks. Nature reviews Molecular cell biology. 2017;18(6):389-401.

66. Lomakin AJ, Lee KC, Han SJ, Bui DA, Davidson M, Mogilner A, Danuser G. Competition for actin between two distinct F-actin networks defines a bistable switch for cell polarization. Nature cell biology. 2015;17(11):1435-45. 
67. Rotty JD, Wu C, Haynes EM, Suarez C, Winkelman JD, Johnson HE, Haugh JM, Kovar DR, Bear JE. Profilin-1 serves as a gatekeeper for actin assembly by Arp2/3dependent and -independent pathways. Developmental cell. 2015;32(1):54-67.

68. Gupton SL, Gertler FB. Filopodia: the fingers that do the walking. Science's STKE : signal transduction knowledge environment. 2007;2007(400):re5.

69. Mallavarapu A, Mitchison T. Regulated actin cytoskeleton assembly at filopodium tips controls their extension and retraction. The Journal of cell biology. 1999;146(5):1097-106.

70. Li F, Higgs HN. The mouse Formin mDial is a potent actin nucleation factor regulated by autoinhibition. Current biology : CB. 2003;13(15):1335-40.

71. Goode BL, Eck MJ. Mechanism and function of formins in the control of actin assembly. Annual review of biochemistry. 2007;76:593-627.

72. Pellegrin S, Mellor H. The Rho family GTPase Rif induces filopodia through mDia2. Current biology : CB. 2005;15(2):129-33.

73. Block J, Breitsprecher D, Kuhn S, Winterhoff M, Kage F, Geffers R, Duwe P, Rohn JL, Baum B, Brakebusch C, Geyer M, Stradal TE, Faix J, Rottner K. FMNL2 drives actin-based protrusion and migration downstream of Cdc42. Current biology : CB. 2012;22(11):1005-12.

74. Young LE, Latario CJ, Higgs HN. Roles for Ena/VASP proteins in FMNL3mediated filopodial assembly. Journal of cell science. 2018;131(21).

75. Lebrand C, Dent EW, Strasser GA, Lanier LM, Krause M, Svitkina TM, Borisy GG, Gertler FB. Critical role of Ena/VASP proteins for filopodia formation in neurons and in function downstream of netrin-1. Neuron. 2004;42(1):37-49.

76. Dwivedy A, Gertler FB, Miller J, Holt CE, Lebrand C. Ena/VASP function in retinal axons is required for terminal arborization but not pathway navigation. Development. 2007;134(11):2137-46.

77. Mejillano MR, Kojima S, Applewhite DA, Gertler FB, Svitkina TM, Borisy GG. Lamellipodial versus filopodial mode of the actin nanomachinery: pivotal role of the filament barbed end. Cell. 2004;118(3):363-73.

78. Urbancic V, Butler R, Richier B, Peter M, Mason J, Livesey FJ, Holt CE, Gallop JL. Filopodyan: An open-source pipeline for the analysis of filopodia. The Journal of cell biology. 2017;216(10):3405-22.

79. Bilancia CG, Winkelman JD, Tsygankov D, Nowotarski SH, Sees JA, Comber K, Evans I, Lakhani V, Wood W, Elston TC, Kovar DR, Peifer M. Enabled negatively regulates diaphanous-driven actin dynamics in vitro and in vivo. Developmental cell. 2014;28(4):394-408.

80. Barzik M, McClain LM, Gupton SL, Gertler FB. Ena/VASP regulates mDia2initiated filopodial length, dynamics, and function. Molecular biology of the cell. 2014;25(17):2604-19.

81. Applewhite DA, Barzik M, Kojima S, Svitkina TM, Gertler FB, Borisy GG. Ena/VASP proteins have an anti-capping independent function in filopodia formation. Molecular biology of the cell. 2007;18(7):2579-91.

82. Menon S, Boyer NP, Winkle CC, McClain LM, Hanlin CC, Pandey D,

Rothenfusser S, Taylor AM, Gupton SL. The E3 Ubiquitin Ligase TRIM9 Is a Filopodia Off Switch Required for Netrin-Dependent Axon Guidance. Developmental cell. 2015;35(6):698-712. 
83. Bohil AB, Robertson BW, Cheney RE. Myosin-X is a molecular motor that functions in filopodia formation. Proceedings of the National Academy of Sciences of the United States of America. 2006;103(33):12411-6.

84. He K, Sakai T, Tsukasaki Y, Watanabe TM, Ikebe M. Myosin X is recruited to nascent focal adhesions at the leading edge and induces multi-cycle filopodial elongation. Scientific reports. 2017;7(1):13685.

85. Disanza A, Bisi S, Winterhoff M, Milanesi F, Ushakov DS, Kast D, Marighetti P, Romet-Lemonne G, Muller HM, Nickel W, Linkner J, Waterschoot D, Ampe C, Cortellino S, Palamidessi A, Dominguez R, Carlier MF, Faix J, Scita G. CDC42 switches IRSp53 from inhibition of actin growth to elongation by clustering of VASP. The EMBO journal. 2013;32(20):2735-50.

86. Kast DJ, Dominguez R. IRSp53 coordinates AMPK and 14-3-3 signaling to regulate filopodia dynamics and directed cell migration. Molecular biology of the cell. 2019;30(11):1285-97.

87. Prevost C, Zhao H, Manzi J, Lemichez E, Lappalainen P, Callan-Jones A, Bassereau P. IRSp53 senses negative membrane curvature and phase separates along membrane tubules. Nature communications. 2015;6:8529.

88. Tsai FC, Bertin A, Bousquet H, Manzi J, Senju Y, Tsai MC, Picas L, MisereyLenkei S, Lappalainen P, Lemichez E, Coudrier E, Bassereau P. Ezrin enrichment on curved membranes requires a specific conformation or interaction with a curvaturesensitive partner. eLife. 2018;7.

89. Dodes Traian MM, Gonzalez Flecha FL, Levi V. Imaging lipid lateral organization in membranes with C-laurdan in a confocal microscope. Journal of lipid research. 2012;53(3):609-16.

90. Zhao H, Michelot A, Koskela EV, Tkach V, Stamou D, Drubin DG, Lappalainen P. Membrane-sculpting BAR domains generate stable lipid microdomains. Cell reports. 2013;4(6):1213-23.

91. Bucki R, Wang YH, Yang C, Kandy SK, Fatunmbi O, Bradley R, Pogoda K, Svitkina T, Radhakrishnan R, Janmey PA. Lateral distribution of phosphatidylinositol 4,5-bisphosphate in membranes regulates formin- and ARP2/3-mediated actin nucleation. The Journal of biological chemistry. 2019;294(12):4704-22.

92. Postema MM, Grega-Larson NE, Meenderink LM, Tyska MJ. PACSIN2dependent apical endocytosis regulates the morphology of epithelial microvilli. Molecular biology of the cell. 2019;30(19):2515-26.

93. Jasnin M, Asano S, Gouin E, Hegerl R, Plitzko JM, Villa E, Cossart P, Baumeister $\mathrm{W}$. Three-dimensional architecture of actin filaments in Listeria monocytogenes comet tails. Proceedings of the National Academy of Sciences of the United States of America. 2013;110(51):20521-6.

94. Vignjevic D, Kojima S, Aratyn Y, Danciu O, Svitkina T, Borisy GG. Role of fascin in filopodial protrusion. The Journal of cell biology. 2006;174(6):863-75.

95. Jaiswal R, Breitsprecher D, Collins A, Correa IR, Jr., Xu MQ, Goode BL. The formin Daam1 and fascin directly collaborate to promote filopodia formation. Current biology : CB. 2013;23(14):1373-9.

96. Okenve-Ramos P, Llimargas M. Fascin links Bt1/FGFR signalling to the actin cytoskeleton during Drosophila tracheal morphogenesis. Development. 2014;141(4):92939. 
97. Tilney LG, Connelly PS, Vranich KA, Shaw MK, Guild GM. Why are two different cross-linkers necessary for actin bundle formation in vivo and what does each cross-link contribute? The Journal of cell biology. 1998;143(1):121-33.

98. Aratyn YS, Schaus TE, Taylor EW, Borisy GG. Intrinsic dynamic behavior of fascin in filopodia. Molecular biology of the cell. 2007;18(10):3928-40.

99. Goh WI, Ahmed S. mDia1-3 in mammalian filopodia. Communicative \& integrative biology. 2012;5(4):340-4.

100. Medeiros NA, Burnette DT, Forscher P. Myosin II functions in actin-bundle turnover in neuronal growth cones. Nature cell biology. 2006;8(3):215-26.

101. Bornschlogl T, Romero S, Vestergaard CL, Joanny JF, Van Nhieu GT, Bassereau P. Filopodial retraction force is generated by cortical actin dynamics and controlled by reversible tethering at the tip. Proceedings of the National Academy of Sciences of the United States of America. 2013;110(47):18928-33.

102. Leijnse N, Oddershede LB, Bendix PM. An updated look at actin dynamics in filopodia. Cytoskeleton. 2015;72(2):71-9.

103. Harker AJ, Katkar HH, Bidone TC, Aydin F, Voth GA, Applewhite DA, Kovar DR. Ena/VASP processive elongation is modulated by avidity on actin filaments bundled by the filopodia cross-linker fascin. Molecular biology of the cell. 2019;30(7):851-62. 104. Breitsprecher D, Koestler SA, Chizhov I, Nemethova M, Mueller J, Goode BL, Small JV, Rottner K, Faix J. Cofilin cooperates with fascin to disassemble filopodial actin filaments. Journal of cell science. 2011;124(Pt 19):3305-18.

105. Ling K, Doughman RL, Firestone AJ, Bunce MW, Anderson RA. Type I gamma phosphatidylinositol phosphate kinase targets and regulates focal adhesions. Nature. 2002;420(6911):89-93.

106. Gallo G, Letourneau PC. Localized sources of neurotrophins initiate axon collateral sprouting. The Journal of neuroscience : the official journal of the Society for Neuroscience. 1998;18(14):5403-14.

107. Ketschek A, Gallo G. Nerve growth factor induces axonal filopodia through localized microdomains of phosphoinositide 3-kinase activity that drive the formation of cytoskeletal precursors to filopodia. The Journal of neuroscience : the official journal of the Society for Neuroscience. 2010;30(36):12185-97.

108. Pickering K, Alves-Silva J, Goberdhan D, Millard TH. Par3/Bazooka and phosphoinositides regulate actin protrusion formation during Drosophila dorsal closure and wound healing. Development. 2013;140(4):800-9.

109. Chang C, Adler CE, Krause M, Clark SG, Gertler FB, Tessier-Lavigne M, Bargmann CI. MIG-10/lamellipodin and AGE-1/PI3K promote axon guidance and outgrowth in response to slit and netrin. Current biology : CB. 2006;16(9):854-62. 110. Plantard L, Arjonen A, Lock JG, Nurani G, Ivaska J, Stromblad S. $\operatorname{PtdIns}(3,4,5) \mathrm{P}(3)$ is a regulator of myosin-X localization and filopodia formation. Journal of cell science. 2010;123(Pt 20):3525-34.

111. Onishi K, Shafer B, Lo C, Tissir F, Goffinet AM, Zou Y. Antagonistic functions of Dishevelleds regulate Frizzled3 endocytosis via filopodia tips in Wnt-mediated growth cone guidance. The Journal of neuroscience : the official journal of the Society for Neuroscience. 2013;33(49):19071-85.

112. Chou AM, Sem KP, Wright GD, Sudhaharan T, Ahmed S. Dynamin1 is a novel target for IRSp53 protein and works with mammalian enabled (Mena) protein and Eps8 
to regulate filopodial dynamics. The Journal of biological chemistry. 2014;289(35):24383-96.

113. Yamada H, Abe T, Satoh A, Okazaki N, Tago S, Kobayashi K, Yoshida Y, Oda Y, Watanabe M, Tomizawa K, Matsui H, Takei K. Stabilization of actin bundles by a dynamin $1 /$ cortactin ring complex is necessary for growth cone filopodia. The Journal of neuroscience : the official journal of the Society for Neuroscience. 2013;33(10):4514-26. 114. Gu C, Yaddanapudi S, Weins A, Osborn T, Reiser J, Pollak M, Hartwig J, Sever S. Direct dynamin-actin interactions regulate the actin cytoskeleton. The EMBO journal. 2010;29(21):3593-606.

115. Park RJ, Shen H, Liu L, Liu X, Ferguson SM, De Camilli P. Dynamin triple knockout cells reveal off target effects of commonly used dynamin inhibitors. Journal of cell science. 2013;126(Pt 22):5305-12.

116. Meyer MP, Smith SJ. Evidence from in vivo imaging that synaptogenesis guides the growth and branching of axonal arbors by two distinct mechanisms. The Journal of neuroscience : the official journal of the Society for Neuroscience. 2006;26(13):3604-14. 117. Feany MB, Buckley KM. The synaptic vesicle protein synaptotagmin promotes formation of filopodia in fibroblasts. Nature. 1993;364(6437):537-40.

118. Johnsson AK, Karlsson R. Synaptotagmin 1 causes phosphatidyl inositol lipiddependent actin remodeling in cultured non-neuronal and neuronal cells. Experimental cell research. 2012;318(2):114-26.

119. Llobet A, Gallop JL, Burden JJE, Camdere G, Chandra P, Vallis Y, Hopkins CR, Lagnado L, McMahon HT. Endophilin drives the fast mode of vesicle retrieval in a ribbon synapse. The Journal of neuroscience : the official journal of the Society for Neuroscience. 2011;31(23):8512-9.

120. Matsubayashi Y, Coulson-Gilmer C, Millard TH. Endocytosis-dependent coordination of multiple actin regulators is required for wound healing. The Journal of cell biology. 2015;210(3):419-33.

121. Masters TA, Buss F. Filopodia formation and endosome clustering induced by mutant plus-end-directed myosin VI. Proceedings of the National Academy of Sciences of the United States of America. 2017;114(7):1595-600.

122. Fischer RS, Lam PY, Huttenlocher A, Waterman CM. Filopodia and focal adhesions: An integrated system driving branching morphogenesis in neuronal pathfinding and angiogenesis. Developmental biology. 2018.

123. Jacquemet G, Baghirov H, Georgiadou M, Sihto H, Peuhu E, Cettour-Janet P, He T, Perala M, Kronqvist P, Joensuu H, Ivaska J. L-type calcium channels regulate filopodia stability and cancer cell invasion downstream of integrin signalling. Nature communications. 2016;7:13297.

124. Sawada Y, Tamada M, Dubin-Thaler BJ, Cherniavskaya O, Sakai R, Tanaka S, Sheetz MP. Force sensing by mechanical extension of the Src family kinase substrate p130Cas. Cell. 2006;127(5):1015-26.

125. Richier B, Inoue Y, Dobramysl U, Friedlander J, Brown NH, Gallop JL. Integrin signaling downregulates filopodia during muscle-tendon attachment. Journal of cell science. 2018;131(16). 